\title{
Trabajadores enfermos: Investigación social compartida y exigencia de derechos humanos
}

\author{
Sick workers: \\ Shared social research and call for Human Rights
}

Daniel Velandia Díaz ${ }^{1}$

\begin{abstract}
Resumen
En este artículo presento características, análisis y desafíos de un proceso de investigación cualitativa que se dio en el estudio sobre organizaciones de trabajadores y ex trabajadores enfermos en Colombia. El estudio fue llevado a cabo mediante un convenio entre el Ministerio de Trabajo de Colombia y la Asociación de Abogados Laboralistas de los Trabajadores (Asolaborales). Para comprender las particularidades, el proceso y los alcances de la investigación, propongo la noción de: "investigación compartida". Como se trata de una reflexión sobre la forma en que se llevó a cabo este estudio de las organizaciones de trabajadores, el análisis tomó la forma de introspección sobre los aspectos metodológicos, es decir, de una mirada hacia el interior de los procesos concretos de investigación. Se hace énfasis en el diseño metodológico, el rol del trabajo de campo, el análisis de los datos y las reflexiones colectivas alrededor de la investigación. Como resultado, el artículo muestra cómo el equipo de investigación conformado mayoritariamente por abogados y abogadas obtuvo un conjunto de aprendizajes, mientras conoció y llevó a cabo la investigación cualitativa. La principal conclusión es que la "investigación compartida" permitió el desarrollo de los objetivos de la Asociación de Abogados Laboralistas de los Trabajadores (Asolaborales), fortaleció el diálogo interdisciplinario y pudo constituirse como una herramienta de trabajo para algunos procesos del derecho.
\end{abstract}

Palabras clave: metodología en ciencias sociales; antropología del trabajo; trabajadores enfermos; derechos económicos, políticos y sociales; derechos humanos; proceso de investigación.

Tipología: Artículo de investigación.

Recibido: 11/09/2017

Evaluado: $26 / 10 / 2018$

Aceptado: 19/11/2018

Disponible en línea: $24 / 12 / 2018$

Como citar este artículo: Velandia Díaz, D. (2019). Trabajadores enfermos: Investigación social compartida y exigencia de derechos humanos. Jangwa Pana, 18 (1), 56-71. Doi: http: http://dx.doi.org/10.21676/16574923.2691

\footnotetext{
${ }^{1}$ Doctor en Antropología. Profesor de la Universidad del Magdalena. ORCID ID: 0000-0001-5523-8479. Correos electrónicos: dvelandia@unimagdalena.edu.co / dvelandiad@unal.edu.co
} 


\begin{abstract}
This article presents characteristics, analysis, and challenges of a qualitative research process that occurred in the study of sick workers' organizations in Colombia. The study was carried out through an agreement between the "Ministerio de Trabajo de Colombia" and the "Asociación de Abogados Laboralistas de los Trabajadores" (Asolaborales). Here, the proposal is about the notion of "shared research", to understand the particularities, process and scope of the research. The analysis shows introspection on the methodological aspects, that is, of a look inside the concrete research processes. Emphasis is placed on methodological design, the role of field work, the analysis of data, and collective reflections around research. As a result, the article discusses how the research team, made up mostly of lawyers, obtained a set of learnings while they met and carried out the qualitative research. The main conclusion is that the "shared investigation" allowed the development of the objectives of the "Asociación de Abogados Laboralistas de los Trabajadores" (Asolaborales), strengthened the interdisciplinary dialogue, and could be constituted as a working tool for some legal processes.
\end{abstract}

Keywords: methodology in social sciences; anthropology of work; sick workers; economic, political and social rights; Human Rights; research process.

\section{Introducción}

$\mathrm{E}^{1}$ Ministerio de Trabajo de Colombia y la Asociación de Abogados Laboralistas de los Trabajadores (Asolaborales) suscribieron en el año 2015 el Convenio Interadministrativo 295 con el objetivo de realizar un diagnóstico detallado sobre la situación de los trabajadores y ex trabajadores enfermos que, en ese momento, se encontraban asociados. Igualmente, el Convenio propuso que las recomendaciones derivadas del análisis apoyasen la toma de decisiones sobre la materia. Este diagnóstico de trabajadores y ex trabajadores enfermos, en el cual se incluyeron también trabajadoras y extrabajadoras, se llevó a cabo durante los años 2015 y 2016.

El diagnóstico de los trabajadores y ex trabajadores enfermos asociados implicó la realización de una investigación socio jurídica amplia compuesta por cuatro áreas: 1 . Análisis crítico de la literatura nacional e internacional producida en el período 2012-2015; 2. Caracterización de las organizaciones de trabajadores enfermos; 3. Caracterización sociodemográfica, patológica, de calificación (evaluación médico-legal de la condición de salud-enfermedad) y de participación de trabajadores y ex trabajadores enfermos asociados; y 4. Análisis técnico-jurídico. Los resultados del diagnóstico se presentaron en el Informe Final en junio de 2016 (Ministerio de Trabajo y Asociación de Abogados Laboralistas de los Trabajadores, 2016).

En este artículo muestro los retos que se presentaron cuando se consideró mi participación en esta investigación bajo el cargo de asesor metodológico para el trabajo de campo. A comienzos del año 2016, cuando recientemente había culminado mi proceso de formación doctoral, recibí la invitación para participar en el Convenio Interadministrativo 295. En ese entonces la vinculación al convenio significó, también, mi acercamiento al fenómeno social de los trabajadores enfermos en Colombia y la interacción con la Asociación de Abogados Laboralistas de los Trabajadores (Asolaborales).

Mis actividades estuvieron relacionadas con el apoyo al director del proyecto y al grupo operativo de la asociación en los aspectos metodológicos de la caracterización. El grupo estuvo conformado por profesionales del derecho, salud, salud pública, antropología y 
administración. Este grupo de veinte personas, compuesto mayoritariamente por abogados y abogadas, tuvo como una de sus funciones la identificación, realización de encuestas y entrevistas a las organizaciones de trabajadores. El grupo se desplazó a los lugares en donde se reúnen los trabajadores y ex trabajadores enfermos organizados (la mayoría de ellos en asociaciones formalizadas) y adelantó el trabajo de investigación cualitativo de la caracterización. Usualmente, a cada lugar se trasladaron dos o tres personas para realizar una jornada de trabajo de campo.

Asimismo, dentro de la ejecución del convenio ocupé un lugar específico que me permitió hacer aportes y conocer una parte de los derroteros de la asociación. Si bien el producto más relevante de mi trabajo fue el análisis de las experiencias colectivas de las organizaciones de trabajadores y ex trabajadores enfermos, quiero detenerme en un conjunto de procesos y subproductos que hicieron posible este resultado final. En gran medida, a continuación, muestro una aproximación a las actividades de investigación a través de la introspección (Woolgar, 1996).

\section{Diseño metodológico}

Junto con la invitación que recibí para participar en la investigación sobre los trabajadores y ex trabajadores enfermos asociados, conocí la propuesta de llevar a cabo, por un lado, un intercambio de habilidades disciplinares con diferentes profesionales y, por otro lado, prever un acercamiento abierto y respetuoso con las personas que iban a formar parte de la investigación como "colaboradores" (trabajadores y ex trabajadores enfermos). Estas previsiones tuvieron que ser consideradas durante toda la investigación y, también, en el momento en el que se llevaron a cabo las actividades preliminares que me fueron solicitadas.

De forma individual y con carácter preparatorio elaboré un par de documentos de trabajo. Primero, un "documento de apoyo metodológico". En este documento sucinto presenté los elementos que serían relevantes indagar junto con los trabajadores $\mathrm{y}$ ex trabajadores enfermos. Cabe señalar que utilicé herramientas para el levantamiento de historias de vida y la indagación de la experiencia de grupos sociales concretos (Godard, 1996). El documento recogió: (A) los parámetros que guiarían la investigación cualitativa sobre la historia de las organizaciones, (B) la forma en la que se realizarían entrevistas y grupos focales, (C) cuatro grandes campos de observación y (D) el procedimiento de análisis de la información.

Las historias de las asociaciones fueron conocidas, en gran medida, a través de las entrevistas y de los grupos focales. Tanto las unas como los otros incluyeron los mismos campos de observación, pero se diferenciaron en las dinámicas de interacción individual o colectiva. Las entrevistas buscaron una aproximación a las experiencias vividas por trabajadores y ex trabajadores, tanto en su actividad laboral individual como dentro de la organización a la que se vincularon. Las experiencias personales se expresaron a través de discursos informales, comentarios, anécdotas, conversaciones y expresiones convencionales que las personas compartieron con el equipo de investigación; con este proceso también pudimos conocer las perspectivas hacia el futuro. El trabajo de investigación tomó como base la entrevista semiestructurada, no-directiva, abierta o informal.

La entrevista semiestructurada que tratamos de seguir durante la investigación fue una relación social comunicativa, similar a una conversación corriente, que permitió el encuentro de las personas con el objetivo de profundizar en el conocimiento sobre las experiencias laborales. Este tipo de entrevista abordó la temática de forma global y permitió descubrir e incorporar el punto de vista y los temas de interés de los entrevistados y las entrevistadas (es deseable que en ciencias sociales haya mayores indagaciones sobre la base de esta postura ético-política). De tal forma, el entrevistado pudo formar un 
discurso propio alrededor de lo que deseábamos conocer. La entrevista semiestructurada, como señalan Díaz-Bravo, Torruco-García, MartínezHernández y Varela-Ruiz:

Se puede definir como una "conversación amistosa" entre informante y entrevistador, convirtiéndose este último en un oidor, alguien que escucha con atención, no impone ni interpretaciones ni respuestas, guiando el curso de la entrevista hacia los temas que a él le interesan (2013, p.164).

Por su parte, los grupos focales consistieron en conversaciones colectivas que nos permitieron acercarnos a la perspectiva de los participantes, un colectivo de trabajadores relativamente homogéneo (Liamputton, 2011). Para el empleo de los grupos focales se aplicaron, inicialmente, las mismas reglas de las entrevistas semiestructuradas. Además, en este tipo de grupos las personas, por lo general, vinculadas a la misma organización o asociación de trabajadores y/o ex trabajadores de una empresa, exploraron y clarificaron su propio punto de vista al conversar con otras personas. Por ejemplo, durante el desarrollo de varios grupos focales, los trabajadores y ex trabajadores presentes conversaron acerca del número real de asociados vinculados y/o activos. En algunos casos, no se sabía claramente si las personas pertenecientes a un sindicato estaban o no en la organización y, en otros, no estaba claro si para ser asociado se requería obligatoriamente una afiliación oficial.

De esta forma, los grupos focales permitieron conocer múltiples interpretaciones sobre varios asuntos y la forma en la que los trabajadores y ex trabajadores argumentaron su posición.

\footnotetext{
${ }^{2}$ Primero, realizar la caracterización de los trabajadores y extrabajadores enfermos asociados en Colombia (Identificar las asociaciones en Colombia; caracterizar los trabajadores y extrabajadores enfermos asociados; conocer los principales aspectos que los llevaron a asociarse; conocer el impacto al interior de las empresas, el estado de sus procesos de calificación; y conocer la morbilidad prevalente). Segundo, conocer el
}

Igualmente, las personas menos participativas pudieron conocer, asumir y compartir opiniones que habían sido aceptadas por el grupo. Además, los grupos implicaron la previsión de la participación decidida del moderador o moderadora para otorgar el tiempo adecuado a las intervenciones y la necesidad de tratar de evitar que hubiese sólo una voz que opacase a las otras. Esta dinámica permitió que surgieran temas u opiniones que los investigadores no podíamos prever, ya que la interacción mostró la forma como las personas entienden y negocian su propia realidad (Krueger, 1998).

Este diseño metodológico buscó ser una conexión entre los objetivos del proyectoconvenio $^{2}$ y las actividades concretas que se llevarían a cabo en el trabajo de campo. Aquí se manifestó la posibilidad de mostrar el camino que llevaría a la Asociación de Abogados Laboralistas de los Trabajadores hacia el cumplimiento de una de las metas del convenio: la caracterización de las organizaciones de trabajadores enfermos. Si bien el diseño metodológico estaba escrito para avanzar en un proceso sistemático de investigación, también podía interpretarse la necesidad de convencer a un conjunto de abogados y abogadas de la posibilidad real de llevar a cabo una investigación cualitativa con trabajo de campo.

El apoyo metodológico no se restringió a mostrar los procesos de selección, recolección, análisis y presentación de información. Para mí fue una notable oportunidad para cautivar y acompañar a un conjunto de profesionales del derecho para que llevaran a cabo actividades diferentes a las que acostumbraban. Me interesó promover la investigación basada en la inmersión en la vida cotidiana de las personas,

cumplimiento o no de los estándares de protección y garantía de derechos relacionados con la prevención, atención y la reparación de los riesgos laborales y comunes. Y tercero: formular recomendaciones para la toma de decisiones sobre la materia, con la intención de ofrecer respuestas adecuadas desde un enfoque de salud pública que atienda esta problemática. 
recolección de información, análisis de datos, comparación y confrontación de conjeturas y presentación de resultados. En este sentido, este tipo de investigación cualitativa sería útil para el convenio $\mathrm{y}$, eventualmente, para la vida profesional de abogados y abogadas.

En segunda instancia, elaboré dos documentos similares entre sí: (1) Consentimiento Informado - Entrevista y (2) Consentimiento Informado Grupo Focal. Se trató de documentos básicos para el desarrollo del trabajo de campo y la interacción entre el grupo operativo de Asolaborales y los trabajadores y extrabajadores enfermos. El diseño de estos consentimientos, en un formato legible y útil, implicó mi acercamiento a modelos preexistentes, sobre todo en el área de la salud, para la atención psicológica o médica. Así logré construir un formato que aclaró la participación libre y voluntaria de las personas en la investigación. Se prescribió que los formatos de los consentimientos fueran revisados y diligenciados antes de comenzar las entrevistas y los grupos focales (aun cuando, algunas veces, se procedió a la firma y la formalización en otro momento durante los encuentros).

Los formatos de los consentimientos se redactaron en primera persona del singular con el objetivo de que cada uno de los trabajadores o extrabajadores enfermos los suscribiera. Una vez las personas conocieran las condiciones de la participación dentro de la investigación, podían decidir si continuaban el proceso o no. Esta previsión implicó que toda la información recogida contara con fuentes verídicas y que las personas tuviesen claro que la investigación se realizaría solo con los datos que ellas quisieran brindar. Una de las especificaciones incluidas dentro de los consentimientos fue:

Los investigadores(as) y moderadores(as) me han dicho que el grupo focal podría ser grabado y tener una duración de dos horas aproximadamente. Se tratarán temas relacionados con la historia laboral de varias personas desde que ingresaron a trabajar, pasando por el momento en que sufrieron la enfermedad hasta el tiempo presente.

El lenguaje utilizado previó la legitimidad y la legalidad de la relación producida en el contexto de la investigación. Este aspecto se hizo evidente porque en la parte baja del documento se dispusieron espacios para la firma tanto del entrevistado o entrevistada como del investigador o investigadora y, además, en caso de haberlos, se podrían incluir las firmas de un representante legal (de la persona entrevistada) o de un testigo. En última instancia, los consentimientos, en la medida en que fueron acuerdos voluntarios, podían ser anulados en cualquier momento si la persona lo deseaba.

De esta forma comenzó mi participación en calidad de antropólogo en el grupo operativo, en el contexto del apoyo metodológico para el levantamiento y análisis de datos. No obstante, teniendo presente que el equipo de trabajo no estaba totalmente preparado para la realización de la investigación cualitativa ni del trabajo de campo, consideré necesario (aunque el director del proyecto y los asociados de Asolaborales también lo habían considerado así desde antes) hacer una presentación sobre la pertinencia de la investigación cualitativa, la entrevista y el grupo focal $y$ algunas reflexiones sobre "saber escuchar". Esto último, como antesala para sensibilizar sobre la disposición personal hacia la investigación social.

Entregué estas herramientas una semana antes del encuentro personal con el grupo operativo de la Asociación de Abogados Laboralistas de los Trabajadores para que fueran revisadas antes de llevar a cabo una presentación formal. A partir de ese momento, esperé los ajustes necesarios y los comentarios a estos documentos de trabajo. $\mathrm{Si}$ bien el director del proyecto y el asesor médico laboral tenían un conocimiento profundo de las metodologías cualitativas, era mi responsabilidad dar credibilidad a este enfoque y lograr que los futuros investigadores tuvieran suficiente motivación y herramientas para llevar a cabo un trabajo con resultados favorables. 


\section{Investigación compartida}

En antropología se han realizado, analizado y conceptualizado procesos de investigación colaborativa (Davies, 1999; Jimeno, 2004; Rappaport, 2008; Tuhiwai, 2007; Vasco, Aranda y Dagua, 1998; cf. Velandia, 2007, 2016). Una parte de los trabajos que mencionan la investigación colaborativa hace énfasis en la producción conjunta de "todo" el proceso de investigación; algunos trabajos incluyen la formulación de las preguntas de investigación, el desarrollo metodológico, las propuestas teóricas, la escritura y la divulgación de los resultados. Quizá lo más destacable de este tipo de investigación sea el hecho de que los compromisos de la investigación vinculan las preocupaciones y/o derroteros de los "sujetos" de investigación con las propuestas teóricometodológicas de quienes investigan. En las investigaciones colaborativas es corriente que los investigadores o investigadoras compartan la identidad de los sujetos o pertenezcan a los grupos sociales sobre los cuales se adelanta la investigación.

Por supuesto, cada investigación en antropología tiene particularidades y no es adecuado definir la "colaboración" de forma taxativa; a su vez, es posible utilizar esta noción para diferentes escenarios de producción de conocimiento. Sin embargo, quisiera anotar que para los fines del presente análisis prefiero utilizar la noción de "investigación compartida" para significar un proceso de investigación conjunto que incluyó diversos actores, combinó varias búsquedas de conocimiento y acción, utilizó varias herramientas para registrar información y analizar datos y, otorgó autonomía a cada una de las actividades y/o destrezas profesionales. En definitiva, la "investigación compartida" valoró positivamente la producción de evidencias sobre las experiencias del sujeto social, evidencias que fueron generadas a partir de diferentes saberes. Todo lo cual apoya la idea de hacer investigación en el marco de un proyecto mayor (y algunas veces tácito) que, en el caso del convenio, fue dar visibilidad a los trabajadores y extrabajadores enfermos asociados.

Mientras se desarrolló la investigación de campo se hicieron simultáneamente otros procesos, entre ellos, el seguimiento a demandas y/o la caracterización socio-demográfica. En este sentido, la visibilidad de los trabajadores enfermos se concibió como posible por la realización de muchas actividades y no solo por la realización del trabajo de campo basado en observación, entrevistas y grupos focales. Por lo cual la investigación consideró con igual valoración cada uno de los siguientes aspectos: la producción de conocimiento, la metodología antropológica, la teorización o la discusión académica. De tal forma considero que el rol de mi perspectiva antropológica dentro de la caracterización fue mayormente de apoyo en tanto que herramienta y saber útil en el proceso de visibilización. Así las cosas, pienso que todas las personas que participamos en la investigación "compartimos" un conjunto de derroteros, mientras la investigación de campo fue un medio parcial para lograrlos.

Ahora bien, los objetivos antropológicos incluyeron diseñar, guiar, analizar, documentar la investigación de campo y, especialmente, recoger la experiencia de trabajadoras y trabajadores que se encontraban enfermos por causa del trabajo que realizaron o continúan realizando. Mis actividades fueron complementarias a las actividades que ya venían realizando otros miembros del equipo de investigación, como, por ejemplo, el análisis socio-jurídico. En la primera jornada, cuando presenté la estrategia de investigación a la Asociación de Abogados Laboralistas de los Trabajadores, pude conocer el equipo de investigación y notar que lograr la empatía con las personas de este grupo sería el factor determinante para continuar con el trabajo.

En este sentido, si bien uno de los principales objetivos prácticos fue brindar herramientas generales para que el equipo pudiera emprender la caracterización cualitativa de cara al logro de 
las metas; para muchas personas de Asolaborales lo más importante fue contar con una persona con la cual desearan trabajar en el futuro. Tuve suerte en este asunto, puesto que algunas personas me manifestaron la intención de continuar trabajando conmigo. A pesar de que yo era un neófito en el proyecto y en la asociación, mis intereses personales sí giraban en torno a las experiencias laborales de la gente y coincidieron con las expectativas de algunas personas del grupo operativo. En términos generales, se podría decir que estábamos preocupados por la situación de la clase-que-vive-del-trabajo (Antunes, 2000).

El equipo de investigación estuvo conformado principalmente por abogados y abogadas que conocían la situación de los trabajadores y extrabajadores enfermos. En la mayoría de los casos el equipo había llevado en el pasado, o llevaba en ese justo momento, el seguimiento de procesos individuales (civiles, penales, jurídicos y/o judiciales), lo que permitía tener un conocimiento de la problemática de los trabajadores enfermos. Además, algunos abogados y abogadas conocían particularidades y luchas de aquellos grupos que se organizaron para reivindicar derechos laborales, económicos, políticos y sociales.

Fue así como se hizo necesario reconocer y aprovechar los conocimientos previos de los profesionales del grupo operativo y fortalecer las habilidades para la recolección y análisis de información cualitativa de carácter colectivo. Si bien dentro de las actividades propias de abogados y abogadas se encontraba la recolección de documentos y la elaboración y revisión de expedientes de un "caso" para su resolución favorable; dentro de la caracterización las tareas diseñadas para el encuentro con los trabajadores y extrabajadores fueron diferentes. Particularmente, la tarea más importante fue el levantamiento de datos nuevos y la subsecuente construcción de un documento. Por lo menos esa fue mi proyección inicial.

\section{El rol en el trabajo de campo}

La investigación buscó un acercamiento de primera mano a las organizaciones de trabajadores o extrabajadores enfermos existentes en el territorio nacional. Sobre la base de la experiencia de la Asociación de Abogados Laboralistas de los Trabajadores (Asolaborales) se revisaron las organizaciones principales y las respectivas empresas para las que trabajan o trabajaron. De esta forma, se planificó visitar quince departamentos: Arauca, Bogotá D.C., Bolívar, Boyacá, Caldas, Cesar, Córdoba, Cundinamarca, Guajira, Huila, Magdalena, Meta, Norte de Santander, Santander y Valle del Cauca. En estos departamentos se logró conocer grupos de trabajadores y extrabajadores vinculados a los sectores productivos: mineroenergético, industria, agricultura, construcción, servicios, entre otros.

Con el propósito de llegar a todos los lugares propuestos en el proyecto, se conformaron grupos pequeños de trabajo que se desplazaron a cada una de las organizaciones y desarrollaron independientemente su propio trabajo de campo. De esta forma se logró llegar a todas las organizaciones previstas y distribuir el trabajo de recolección de información. Mi participación tuvo el objetivo de acompañar a cada uno de los grupos en una primera jornada de trabajo de campo para que, posteriormente, desarrollara jornadas de recolección de información con el conocimiento y la práctica necesarios para llevar a cabo la metodología.

En la primera jornada de trabajo con cada uno de los seis grupos pequeños (conformados por dos, tres o cuatro personas) se generó un proceso de trabajo de campo propio que dependió mucho de las capacidades e iniciativa de cada uno de los integrantes. Mi participación, no obstante, se enfocó en la realización de tareas similares para todos los lugares. En primer lugar, consideré que mi responsabilidad estaría en establecer los términos de "la relación de investigación" con las personas con las que iríamos a conversar. En este sentido, mi aproximación a los lugares de 
observación implicó hacer previsiones respecto a la presentación explícita del convenio, de los consentimientos, de las actividades a realizar y de las expectativas de la Asolaborales respecto al proceso en su conjunto.

En los primeros momentos, cuando se organizaron las actividades preliminares al interior de los grupos pequeños de Asolaborales, primó siempre la pragmática de conseguir la interacción efectiva con el grupo de trabajadores o ex trabajadores enfermos. En la medida en que los abogados y abogadas conocían a algunas personas claves, la tendencia fue tratar de conversar con la mayor parte de estos conocidos. Casi siempre, en los primeros encuentros con las personas de las organizaciones, se dio paso a las presentaciones personales, a la contextualización general del lugar y a comentarios acerca de las particularidades de la reunión.

En todas estas experiencias inaugurales de trabajo de campo con cada uno de los grupos pequeños se previó la realización de, por lo menos, una jornada de entrevistas con varias personas y un grupo focal. Cada equipo de Asolaborales, junto con los trabajadores o ex trabajadores enfermos de cada departamento, acordamos la realización de estas jornadas y, de esta forma, la primera tarea que cumplimos de forma práctica fue la planeación de una agenda común. En algunos casos, esta agenda implicó el desplazamiento del grupo a varios lugares para tener contacto directo con las personas. Por ejemplo, cuando estuvimos en la ciudad de Manizales conocimos el área de influencia de una empresa y los barrios circunvecinos en donde viven muchos de los trabajadores y ex trabajadores enfermos y sus hogares.

Así fue como, simultáneamente a la búsqueda de la información por medio de entrevistas y grupos focales, se logró conocer asuntos relevantes de la situación general de los trabajadores en las empresas, especialmente, la interdependencia de la situación de salud de los trabajadores, las familias y los roles de género (Creed, 2000); asunto que, a su vez, estaba asociado a la idea general de trabajar de forma continua para el sostenimiento de un hogar (Besen, 2007). Este tipo de información estuvo presente tanto en las observaciones que pudimos realizar desde el grupo de investigadores, como en las reflexiones $\mathrm{y}$ conversaciones que tuvieron lugar en el contexto del trabajo de campo.

Posteriormente, en el desarrollo de los grupos focales siempre fue importante tratar de conocer el punto de vista de muchas personas, incluso los posibles desacuerdos que existiesen al interior del grupo. La mayor parte del tiempo los grupos focales fueron sesiones en las cuales las personas trataron de complementar información con el objetivo de profundizar en las situaciones desfavorables para los trabajadores en las empresas. En este sentido, la moderación de los grupos, que yo mismo realicé, buscó encontrar aquellos acuerdos generales sobre la percepción y sobre los hechos significativos en relación con la situación de trabajo y enfermedad común al grupo.

En la mayoría de los grupos focales fue difícil conocer perspectivas diversas de asociados o de facciones de asociados. Los liderazgos prevalecieron y los asociados individuales (o beneficiarios) pocas veces pusieron de presente puntos de vista distintos a los establecidos por las personas más influyentes en cada lugar. Si bien se trató de la puesta en escena de relaciones de poder basadas en el conocimiento, la experiencia o el liderazgo; para nosotros, como grupo de investigadores, fue ciertamente claro que no había divisiones mayores al interior de cada asociación y los trabajadores y ex trabajadores enfermos. En este sentido, el proceso de investigación apoyó la reflexión interna de las asociaciones y de cada trabajador o ex trabajador que participó en las entrevistas y grupos focales.

El apoyo de Asolaborales se percibió, en general, como positivo para la situación de las organizaciones locales. De la misma forma, la realización de los grupos focales fue edificante 
para Asolaborales puesto que permitió obtener información detallada sobre los procesos históricos, administrativos y sociales en relación con las empresas y la forma en que estas gestionan la salud de las personas. Se trató de conocer explícitamente un conjunto de datos sobre la organización de los trabajadores enfermos. El grupo de Asolaborales, o cada abogado o abogada por su cuenta, conocía casos individuales y tenía observaciones generales sobre la historia de las organizaciones antes de la investigación, pero no se había detenido a recoger la experiencia y las reflexiones de estos grupos.

Todo lo cual afirma la idea de que los miembros de cada asociación pudieron profundizar $\mathrm{y}$ analizar las interpretaciones propias sobre la experiencia compartida. Asolaborales propició, en el momento de la investigación cualitativa, la puesta en marcha de este proceso de reflexividad. Como menciona Islam: “...cultures contain, more or less visibly, spaces for critique and reflexivity, without which informants would not be able to interpret or narrate their own cultures" $\left(2015\right.$, p.247) ${ }^{3}$.

Es necesario señalar que, no obstante, algunas organizaciones han vivido procesos históricos de unión o división de grupos de trabajadores o ex trabajadores que se relacionan con sindicatos o grupos de interés específico (v.g. viudas de extrabajadores fallecidos a causa de enfermedades laborales). De la misma forma, los vínculos entre los asociados se sostienen necesariamente por un proceso de reflexión e identificación (v.g. trabajadoras que, a pesar de haber sido despedidas, reivindincan derechos laborales vulnerados en una empresa y se vinculan a la asociación de empleadas activas). Estos asuntos se hicieron visibles durante el trabajo de campo aun cuando no fueron un tema central para la indagación. En términos generales, durante la investigación se presentaron procesos de reflexión propia dentro

\footnotetext{
${ }^{3}$ En traducción libre: "las culturas tienen, de forma más o menos visible, espacios para la crítica y la reflexividad,
}

de cada organización y/o asociación establecida formalmente.

Aparecieron temas recurrentes tales como la indiferencia de algunos trabajadores a los accidentes y a la prevención, especialmente antes de enfermarse; reflexiones en torno al control de las empresas para evitar que los trabajadores y trabajadoras se organicen y reivindiquen sus derechos; o la disposición general de los trabajadores al sobreesfuerzo propio para obtener mayores ingresos. En algún momento de la investigación para mí fue evidente que, tal como lo señaló Palermo para el caso de los trabajadores petroleros en Argentina: "La interiorización de la disciplina fabril hace propia la conducta inevitable de "resolver sin preguntar" y al mismo tiempo de "no interrumpir el trabajo" si ocurre un accidente" (2017, p.90).

A su vez, con cada uno de los grupos pequeños de investigación se presentó un proceso de organización del trabajo que permitió llevar a cabo observaciones, entrevistas, grupos focales y hacer el levantamiento de la información. La apertura para escuchar asuntos propios de los trabajadores fue una herramienta del trabajo de campo, basada en entrevistas semiestructuradas y grupos focales, que todo el Grupo Operativo conoció y comenzó a utilizar para adelantar la investigación.

Quizá lo más relevante de estas experiencias de campo con cada uno de los grupos pequeños en los que participé fue la posibilidad de mostrar cómo se podía desarrollar el trabajo de campo concreto para obtener los resultados tangibles con los trabajadores y/o extrabajadores. Durante los meses de enero y febrero de 2016 acompañé directamente el proceso de recolección de información en las ciudades y/o municipios de Barrancabermeja, Cartagena, Cúcuta, Manizales, San Alberto, Ubaté y Santa Marta. Se destacó el hecho de que los miembros del equipo

sin los cuales los informantes no serían capaces de interpretar o narrar sus propias culturas". 
de investigación pudieron notar que las actividades de investigación son bien distintas a las actividades de defensa legal.

Además, muchos de los profesionales de la Asolaborales hicieron aportes y/o plantearon alternativas dentro del proceso de interacción con las personas entrevistadas. Me permito señalar las sugerencias que presentó Carlos, uno de los abogados con quien me desplacé a Manizales, después de que yo había indicado los cuatro momentos de descripción de la experiencia a los trabajadores enfermos asociados en uno de los primeros encuentros:

Una pregunta, Daniel: ¿no vamos a hacer un registro cronológico? Para que no se escape nada. Porque como lo estamos haciendo, está bien, pero no es cronológico. Se pueden quedar cosas por fuera y, atar los cabos después, puede ser más difícil.

Aparte de los comentarios que pude hacer a esta sugerencia, basados en el hecho de querer conocer de forma amplia la experiencia de las personas, esta intervención me mostró que el grupo operativo tenía una actitud crítica frente a la investigación cualitativa planteada. De hecho, supe que sería necesario presentar las fortalezas de la metodología y los alcances de esta de forma explícita y práctica. Frente a esta situación, me pareció pertinente continuar el proceso de las entrevistas y grupos focales con pragmatismo para mostrar a los colegas del grupo operativo la forma de llevar a cabo el proceso.

Por supuesto, la experticia profesional de los abogados y abogadas nunca estuvo en duda. Desde mi rol como antropólogo traté de acompañar el proceso de investigación y presentar formas (alternativas) de conocer la trayectoria laboral de las organizaciones de trabajadores y extrabajadores enfermos. En este sentido, una síntesis apropiada puede ser que el trabajo de campo dentro de la investigación cualitativa dio elementos para entender procesos colectivos y amplió la idea que Asolaborales tenía de un conjunto de casos individuales por resolver. De hecho, la investigación compartida se basó particularmente en incluir las apreciaciones de varios colegas.

Dentro de los aprendizajes o particularidades del trabajo de campo puedo señalar algunas. En el contexto de lo más pragmático, la recolección de información a través de entrevistas y grupos focales fue seguida por la grabación, la transcripción y la organización de la información. Sin embargo, para algunos investigadores de los grupos fue mejor levantar la información y organizarla directamente. En otras palabras, algunos abogados y abogadas llevaron a cabo los encuentros con las organizaciones y registraron simultáneamente la información en archivos de computador. Para estos investigadores significó una reducción de tiempo de trabajo y la posibilidad de acopiar toda la información necesaria.

También algunos abogados encontraron en el trabajo de campo de la investigación cualitativa la oportunidad para registrar hechos vividos por las organizaciones, que no habían sido tenidos en cuenta anteriormente. Específicamente, se pudieron llevar a cabo grabaciones en video de algunas entrevistas y grupos focales como soporte y memoria de la historia de tales organizaciones; muchas veces conocimos la historia laboral de varias personas en múltiples empresas y con modalidades contractuales diversas. Si bien esto nos permitió conocer el ejercicio continuado de los oficios y las actividades comunes a un conjunto de personas (v.g. industria petrolera), también hizo visible que "el mismo contrato salarial se ha convertido en una fuente de fragmentación y precariedad, y no de homogeneidad y seguridad sociales..." (Wacquant, 2007, p.175).

De hecho, durante el desarrollo del trabajo de campo se obtuvo información de diferente naturaleza, todo lo cual mostró que fue positivo aprovechar los recursos y esfuerzos para llegar a los grupos de trabajadores y extrabajadores asociados. Como se puede apreciar, el desarrollo del convenio posibilitó que los abogados y 
abogadas tuvieran un acercamiento diferente a la situación colectiva de las organizaciones y emprendieran tareas complementarias a la defensa y seguimiento a casos y/o demandas.

Finalmente, mi participación al interior de cada uno de los subgrupos del grupo operativo fue posible por la incorporación de aprendizajes y sinergias que nutrieron el trabajo de campo. Si bien durante las actividades en los diferentes municipios logré un aprendizaje propio sobre la situación de los trabajadores y extrabajadores enfermos; también es cierto que mi rol principal, como guía para los grupos de investigación en el contexto del trabajo de campo, se basó en brindar herramientas y dar cabida al desempeño propio de cada investigador. En esta medida, la "investigación compartida" aparejó la necesidad de adelantar procesos significativos para los participantes; procesos que a su vez pudieron ser replicados por los nuevos investigadores en otras organizaciones y municipios que abordaron posteriormente durante el desarrollo del convenio.

\section{Análisis de los datos}

Las actividades conjuntas para llevar a cabo el trabajo de campo implicaron la necesidad y posibilidad de acopiar de forma confiable los datos y proceder al análisis. La previsión en este sentido fue doble. Por una parte, se grabaron todas las entrevistas y grupos focales; valga señalar que esto se realizó con el consentimiento de las personas. Las grabaciones fueron transcritas posteriormente a la realización de los encuentros. Después de tener un conjunto de seis o más grabaciones se enviaron a una persona contratada para la transcripción; en el transcurso de una o dos semanas, las transcripciones en formato electrónico fueron entregadas para el análisis. De esta forma, muy rápidamente se tuvo a disposición un conjunto de datos para proceder a revisión. De hecho, fue posible conocer el estado de avance de algunos grupos pequeños o subgrupos de investigación antes de que yo mismo hubiese llevado a cabo la primera salida de campo con otros.
Además del flujo de la información disponible para hacer los análisis, también se presentaron escenarios emergentes y propios al grupo de Asolaborales para el análisis durante el desarrollo de las entrevistas y grupos focales. En algunas ocasiones fue insistente la pregunta por la utilidad concreta de los datos obtenidos durante el trabajo de campo. Esta inquietud se hizo presente, por lo general, cuando hubo mucho trabajo de recolección de información que mostraba detalles, pero no se podía convertir en prueba (legal). De la misma forma, fue un gran reto para algunos pocos abogados sostener el proceso de entrevista abierta con trabajadores y extrabajadores, especialmente porque esta actividad implicó horas de conversación que no parecían lo suficientemente "científicas".

Hacer una entrevista o guiar un grupo focal no fue siempre una actividad fácil para los abogados y las abogadas de Asolaborales. Sin lugar a dudas, la primera experiencia en este campo de trabajo no es necesariamente cómoda para todas las personas. Por ejemplo, la propuesta de realizar simultáneamente las entrevistas (o grupo focal) junto con la escritura (inscripción) de la información en un computador parecía apoyar la facilidad y disponibilidad inmediata de los datos. No obstante, yo señalé la importancia de lograr "encontrarnos" con las personas, asunto que se hubiese visto comprometido si el computador se encontrase en medio de las personas. Utilizar una grabadora siempre fue una opción más aceptable para registrar los encuentros.

En el terreno del trabajo de campo no siempre se privilegia la pragmática, antes bien, es relevante contar con una interacción adecuada con las personas para lograr abordar los temas de forma franca. Este hecho fue remarcado por Bibiana, una de las abogadas con quien visitamos una asociación en Cúcuta. Ella, en la medida que progresábamos con las entrevistas, pudo conocer mejor los procesos legales que tenía en ese momento. Además de la cantidad de información pertinente que se obtuvo en estas 
entrevistas y grupos focales, ella me comentó que, en adelante, utilizaría estas técnicas para documentar de mejor forma las peticiones de sus defendidos. Esta valoración positiva del proceso de investigación que seguimos, por parte de una abogada, mostró que dentro de la investigación compartida es posible construir estrategias de trabajo dirigidas hacia la visibilidad de los grupos sociales con los cuales se está interactuando.

Conocer la perspectiva de las personas de primera mano y recolectar un conjunto comparable de datos puede convertirse en una herramienta de trabajo potente para que los abogados y abogadas fortalezcan las actividades que les son propias. Esta herramienta puede coadyuvar en la comprensión de las experiencias vividas por las personas y en la búsqueda de la garantía los derechos de los trabajadores y extrabajadores enfermos. Ahora bien, quiero mostrar los (tipos de) datos que fueron centrales dentro de la investigación y algunas reflexiones sobre cómo se llevó a cabo el registro.

Me pareció necesario formalizar la estructura de la información recolectada. Primero, debido a la gran cantidad de encuentros previstos con varios grupos de trabajadores y extrabajadores enfermos y, segundo, porque la realización de entrevistas y grupos focales involucró diferentes subgrupos de investigación. Por su puesto, la organización de la información es una consideración básica para llevar a cabo cualquier investigación. Con estos asuntos en mente diseñé una tabla en Excel denominada: “Clasificación Entrevistas o Grupos". En ella se consignaron algunos datos que fueron comparados y puestos de presente en el informe final.

El archivo contenía una hoja para diligenciar espacios en blanco con la información de una entrevista o de un grupo focal. Este archivo fue enviado a cada uno de los integrantes de los subgrupos operativos que hizo trabajo de campo, con el propósito de que fuera diligenciada posteriormente al encuentro con los trabajadores y extrabajadores enfermos. En esta hoja de cálculo se propuso diligenciar toda la información recogida en las entrevistas y los grupos focales de forma uniforme, señalando varias categorías. La indicación para todo el grupo consistió en diligenciar los campos, incluido un espacio para observaciones donde se podía añadir información que no estaba considerada en las categorías definidas. Igualmente, podrían quedar varios espacios vacíos.

En principio, se encontraban los datos del encuentro mismo que sirvieron como identificación básica:

- Entrevistador(es), entrevistadora(s) o monitores:

- Grupo focal (participantes):

- Entrevista (entrevistado o entrevistada):

- Fecha:

- Lugar:

- Horas inicial y final:

- Asociación:

Este registro básico del encuentro permitió hacer énfasis en el nombre completo de las asociaciones de trabajadores y extrabajadores enfermos, así como conocer los lugares de las reuniones. Posteriormente, la hoja se dividió en cinco partes que reiteraron los asuntos importantes de las entrevistas y grupos focales. La primera parte denominada "Trabajo previo (antes de la enfermedad o accidente)" tuvo dos temas: 1. Caracterización y percepción y 2. El enfoque de la empresa el tema de la salud y la prevención. Esta primera parte fue clave para que las personas entrevistadas ubicaran su experiencia de trabajo a largo plazo y organizaran el discurso.

También cabe resaltar el hecho de que muchas medidas de seguridad y protección en el trabajo son débiles, tanto como el reconocimiento a las condiciones laborales desfavorables de muchas actividades, por ejemplo, la minería. Igualmente, trabajadores y extrabajadores enfermos señalaron de forma recurrente la realización insuficiente de capacitaciones por parte de las 
empresas. Para los fines de este artículo destaco que para algunos abogados y abogadas fue un espacio extraño porque no se refería al tema que nos convocaba inicialmente. Sin embargo, conocer las percepciones de las personas sobre las empresas, sobre las actividades y sobre las medidas de seguridad y salud en el trabajo (existentes o no) fue, muchas veces, un asunto que permitió dar contexto a las prácticas laborales en el momento histórico realmente vivido. Así fue posible evadir anacronismos: las prácticas en seguridad y salud en el trabajo han cambiado con el tiempo.

La segunda parte de la hoja para diligenciar se llamó "Enfermedad o accidente". En este apartado se buscó conocer cinco aspectos y la relación con cinco actores así: 1. Descripción del caso (trabajador/a), 2. Percepción social de "esta" enfermedad (sociedad, vecindario, familia), 3. Actitudes y respuestas de la empresa (empleador), 4. Actitudes y respuestas de EPS (Entidad Prestadora de Salud) y 5. Actitudes y respuestas de ARL (Administradora de Riesgos Laborales). Si bien se previó que las personas presentaran su caso o experiencia traumática de forma abierta y propia, aquí se pudo observar la necesidad de conversar con las personas a partir de algunas observaciones, preguntas $y$ conocimientos elaborados por ramas del derecho y de la medicina. Aquí se hizo evidente la necesidad de contar con grupos interdisciplinarios, tanto como la potencialidad de conocer asuntos de otras especialidades por parte de cada profesional.

Estuvo presente en esta parte de la información recolectada que los trabajadores y extrabajadores enfermos no sólo conocen muy bien su propio caso, sino que también expresan con mucho detalle lo ocurrido. Esta situación, en última instancia, muestra que los sucesos ocurrieron realmente $\mathrm{y}$, además, que los han relatado con anterioridad a otras personas. Fueron frecuentes las alusiones a enfermedades que inicialmente yo mismo desconocía (v.g. epicondilitis), tanto como a palabras relacionadas con las acciones de las empresas luego de la ocurrencia de un accidente o registrar la enfermedad de algún trabajador o trabajadora, especialmente: incidente y reubicación.

En la medida que este fue el espacio para registrar los datos relacionados con el momento mismo del accidente o enfermedad de las personas, la información acerca de las reacciones de las empresas, Entidades Prestadoras de Salud (EPS) y Administradoras de Riesgos Laborales (ARL), fue de gran importancia para abogados y abogadas. Se podría decir que mucha información consignada a través de los relatos de las personas sirvió, adicionalmente, para la documentación de casos concretos.

La tercera sección de la hoja para el análisis de datos fue: "Momento crítico (asumir la condición de trabajador enfermo)". En este apartado se consignó información relacionada con: 1. Nueva relación laboral o no-laboral, 2. Actitud de la familia, 3. Actitud de los compañeros y compañeras, 4. Actitud de la empresa, 5. Procesos individuales y 6. Solicitudes formales. Este apartado se propuso con el objeto de conocer la experiencia individual e inmediata de cada persona luego de conocer que se encontraba enferma o debía continuar un proceso posterior al accidente.

Si bien el interés fue conocer la forma en que el contexto social y cada persona (enferma) asumió la condición de enfermedad, también fue notoria la existencia de asuntos recurrentes y dolorosos que sufrieron muchas personas. Estuvo presente la existencia de acoso laboral por parte de las empresas y de otros trabajadores, así como el recuento de situaciones de discriminación y rechazo por parte de familiares y amigos. En el trabajo de campo y en la realización de entrevistas y grupos focales fue útil la noción de "momento crítico" puesto que marcó una temporalidad común para trabajadores y extrabajadores enfermos. Con esta noción se mostraron las consecuencias sociales de la situación de enfermedad ocasionada por las actividades laborales $\mathrm{y}$, muchas veces, las 
circunstancias que llevaron a las personas a participar en una organización colectiva.

La cuarta sección, denominada "Asociación", hizo referencia a los procesos sociales vividos en el contexto de la organización colectiva. Para la caracterización de las asociaciones u organizaciones se tuvo en cuenta la siguiente información: 1. Historia y caracterización, 2. Organigrama y funcionamiento, 3. Actores con los que se confronta, 4 . Instituciones a las que se solicita, 5. Actores aliados, 6. Logros y 7. Dificultades. Para Asolaborales y, para el desarrollo del convenio, esta sección aportó los elementos centrales para conocer la forma en que trabajadores y extrabajadores enfermos se organizaron. Es seguro que para llegar a conocer a cada asociación u organización es necesario un acercamiento más profundo que complemente las entrevistas y los grupos focales.

Finalmente, la última sección se denominó "Observaciones". En esta sección se pudieron conocer múltiples apreciaciones que por diversas razones no se incluyeron en los anteriores apartados. En una de las hojas diligenciadas se puede leer: "La Asociación se ha convertido en un apoyo moral y familiar para sus asociados, espacio de terapia para los afiliados, un espacio de apoyo y de asesoría para los afiliados". Este tipo de observaciones mostró que las asociaciones son el espacio social privilegiado para que trabajadores $y$ extrabajadores enfermos no solo den trámite a sus procesos legales, sino que reciban el apoyo emocional que todas las personas requieren luego de sufrir una enfermedad laboral. Incluso, se podría decir que la asociación es un escenario primario donde se genera salud.

Al terminar el trabajo de campo se pudo notar que algunos subgrupos de investigación enviaron las grabaciones, pero no las hojas de clasificación diligenciadas; de esta forma, solo pudimos disponer de las correspondientes transcripciones. En otras palabras, las hojas de Excel no fueron diligenciadas para la totalidad de las entrevistas y grupos focales. En definitiva, una gran parte de la información que se pudo analizar estuvo soportada solamente en transcripciones de las grabaciones. Este hecho mostró que el proceso de análisis no pudo ser uniforme, por lo que fue conveniente hacer el trabajo de revisión de datos "en crudo" a partir de las transcripciones.

El análisis de los datos requirió que yo revisase la documentación existente tanto en las transcripciones numerosas como en las hojas "Clasificación Entrevistas o Grupos", un poco más escasas. En el informe final presenté los siguientes apartados que dieron cuenta del análisis: Metodología de la investigación, Enfermedades presentes en el trabajo, Sistema de seguridad social, Asociaciones de trabajadores y extrabajadores enfermos, Trayectorias de trabajadores enfermos, Conclusiones y Recomendaciones. Como se puede apreciar en la configuración de este índice, las pautas que se siguieron para recolectar la información y clasificar las entrevistas y grupos focales no son idénticas. Por supuesto, el análisis permitió presentar los resultados de forma que la exposición caracterizara al conjunto de trabajadores y extrabajadores enfermos. Por esta razón, comencé con los apartados de la metodología y de las enfermedades y dejé, en un apartado final, las trayectorias de las personas.

\section{Conclusiones}

Bajo la noción de "investigación compartida" es posible definir la investigación cualitativa que se dio en el contexto del convenio interinstitucional entre el Ministerio de Trabajo y Asolaborales. La investigación compartida incorporó la reflexividad sobre el quehacer de las actividades del trabajo de campo y el reconocimiento de procesos y actores que entran en relación con la investigación; actores que, a su vez, hacen otras cosas o están interesados en otros asuntos. Esta experiencia de investigación fue posible por el aporte que realizaron muchas personas desde diferentes experticias y labores. De tal forma, la reflexividad en este escenario compartido 
implicó tanto la interacción interdisciplinaria, como la reflexión de trabajadores y extrabajadores sobre los asuntos relacionados con el trabajo, el empleo, la salud, el sistema de seguridad social, las representaciones sociales sobre el trabajo y la enfermedad, etc.

Pienso que el desarrollo del proyecto fue un desafío para los abogados y abogadas de la asociación por varias razones. Primero, porque eran los abogados asociados y las abogadas asociadas quienes estaban comprometidos con la investigación y con la situación de los trabajadores y extrabajadores enfermos, asunto que les exigía mostrar resultados importantes aun cuando ellos no fueran investigadores de profesión. Segundo, porque las tareas pensadas para llevar a cabo no formaban parte de las habilidades que abogados y abogadas hubiesen aprendido anteriormente. Por ejemplo, hacer entrevistas y/o moderar un grupo focal son actividades bien distintas del seguimiento de una demanda y la revisión de la jurisprudencia.

A su vez, dentro de la investigación compartida fue posible que abogados y abogadas reconociesen la realidad de trabajadores y extrabajadores enfermos más allá de la actividad profesional propia de "llevar un caso". La sensibilización para llevar a cabo investigación a través de entrevistas, grupos focales y observación mostró la importancia de compartir diferentes saberes profesionales para llegar al objetivo común de visibilizar las asociaciones de trabajadores y extrabajadores enfermos. Es posible que la visibilidad de sectores sociales hacia la sociedad nacional en su conjunto demande, primero, el reconocimiento de las experiencias de los grupos sociales contadas por ellos mismos. El grupo de Asolaborales pudo ser sensible al conocer la experiencia de muchos grupos de trabajadores de primera mano $\mathrm{y}$, quizás por algunos momentos, colocó entre paréntesis y repensó la práctica profesional del derecho.

Igualmente, uno de los mayores logros que se hizo evidente con el convenio fue el encuentro efectivo de Asolaborales con las asociaciones y organizaciones de trabajadores y extrabajadores enfermos. Si, por una parte, he mencionado la relación existente entre los abogados y las abogadas con trabajadores y extrabajadores en calidad de demandantes individuales; por otra parte, el trabajo de campo permitió cambiar por un momento los roles de abogado-cliente, para abrir paso al "encuentro" que permitió conocer la experiencia conjunta vivida alrededor de la experiencia de la enfermedad en el trabajo. Todo lo cual apoya el mejoramiento de la comunicación con los trabajadores enfermos y las trabajadoras enfermas.

En un contexto más restringido, pero no menos importante, el desarrollo de la investigación permitió también cualificar la comunicación entre el colectivo de los abogados, los investigadores del área de la salud pública y los colaboradores del área de la investigación cualitativa. Particularmente, creo que se pudo apreciar el aporte de cada disciplina para el objetivo común de visibilizar las asociaciones de trabajadores y extrabajadores enfermos. El trabajo de campo y la apertura a la experiencia de los otros pudo verse reflejada en los resultados de la investigación. Claro está, esta es una previsión que tenían tanto el director como las instituciones que fomentaron el desarrollo del Convenio.

Sin lugar a duda, mi participación en el convenio en un grupo interdisciplinario implicó que mis aportes debían ser insumo para obtener los mejores resultados posibles. En esa medida, llevar a cabo la caracterización junto con las personas de Asolaborales fue una oportunidad para promover el desempeño de los abogados y abogadas en el terreno de la defensa de los derechos. De la misma forma, esta interacción de profesionales del derecho, salud, salud pública, antropología y administración, podría ser revisada a la luz de la reflexión sobre las apuestas epistemológicas y prácticas de todos los implicados. 
Las actividades de investigación fueron desarrolladas con el fin de cumplir los objetivos propuestos, pero para mi sorpresa, se presentaron efectos positivos más allá de los resultados esperados. La entrevista fue una herramienta de trabajo útil para adelantar la caracterización y, al mismo tiempo, se convirtió en una herramienta para fortalecer la comprensión de los casos. Es mi deseo que la disposición para escuchar a los trabajadores y trabajadoras sea, en el futuro, una herramienta que permita lograr mejores resultados al momento de la argumentación jurídica.

\section{Agradecimientos}

Agradezco al profesor Jairo Ernesto Luna García del Doctorado en Salud Pública de la Universidad Nacional de Colombia por convocarme para participar en esta investigación. Igualmente, agradezco al profesor Mauricio Torres Tovar por el acompañamiento y los comentarios oportunos durante el proceso de investigación. De manera especial agradezco a los miembros de Asolaborales por brindarme la oportunidad de conocer su admirable labor y por permitirme acompañarlos en el proceso de la investigación. Particularmente debo mi gratitud a Ricardo Ruíz, Cándida Parales, Nancy Rey, Arturo Portilla, Bibiana Parra, Lida Melo, Manuel Novoa, Carlos Dussan, Dasney López, Juddy Calderón, Orlando Pineda, Beatriz Moreno y Rafael Suárez.

Además, debo agradecer a los trabajadores organizados que hicieron aportes a la investigación. En primer lugar, porque me permitieron conocer, a partir de las experiencias que han vivido, los efectos más dolorosos de la producción y del trabajo en las personas. Es decir, me mostraron cómo la enfermedad está relacionada con las condiciones del trabajo existentes en las empresas y con la necesidad ineludible de trabajar que tienen las personas. Igualmente, agradezco a muchas personas que estuvieron atentas al desarrollo de la investigación y apoyaron el trabajo de campo, particularmente a Carlos Julio Castro, Jorge
García, Jorge Noriega Soto, Jaime Jiménez, Moisés Padilla y Aníbal Pérez. Sin este grupo de personas que tuve la oportunidad de conocer $y$, de quienes recibí el apoyo pertinente para lograr el levantamiento de la información de la investigación, hubiese sido imposible llevar a buen término la cualificación de las organizaciones de trabajadores y extrabajadores enfermos.

\section{Referencias}

Antunes, R. (2000). ¿Adiós al trabajo? Ensayo sobre las metamorfosis y el rol central del mundo el trabajo. Bogotá, Colombia: Antídoto.

Besen, Y. (2007). Masculinities at work. Equal Opportunities International. 26(3), 256260.

Creed, G. W. (2000). Family Values and Domestic Economies. Annual Review of Anthropology. 29. pp. 329-55.

Davies, C. A. (1999). Reflexive ethnography. A guide to researching selves and others. New York, United States: Routledge.

Díaz-Bravo, L., Torruco-García, U., MartínezHernández, M., y Varela-Ruiz, M. (2013). La entrevista, recurso flexible y dinámico. Investigación en Educación Médica [en línea], 2(7), 162-167.

Godard, F. (1996). El debate y la práctica sobre el uso de las historias de vida en las ciencias sociales. Cuadernos del Centro de Investigaciones sobre Dinámica Social. Bogotá, Colombia: Universidad Externado de Colombia. II (1). pp. 5-50.

Islam, G. (2015). Practitioners as Theorists: Para-ethnography and the Collaborative Study of Contemporary Organizations. Organizational Research Methods, 18(2), 231-251. 
Jimeno, M. (2004). La vocación crítica de la antropología latinoamericana. Maguaré, $18,33-58$.

Krueger, R. A. (1998). Developing questions for focus group. Thousand Oaks, United States: Sage.

Liamputton, P. (2011). Focus Group Methodology: Principles and practice. Los Angeles, United States: Sage.

Ministerio de Trabajo y Asociación de Abogado s Laboralistas de los Trabajadores (2016). Informe Final, Convenio interadministrativo 295 de 2015: Caracterización de los trabajadores y extrabajadores enfermos asociados en Colombia. Documento de trabajo, $140 \mathrm{pp}$.

Palermo, H. (2017). La producción de la masculinidad en el trabajo petrolero. Buenos Aires, Argentina: Biblos.

Rappaport, J. (2008). Beyond participant observation: collaborative ethnography as theoretical innovation. Collaborative Anthropologies. 1. pp. 1-31.

Tuhiwai S. L. (2007). Decolonizing methodologies. Research and indige-nous peoples. New York, United States: Zed Books y University of Otago Press.

Vasco, L.G., Aranda, M., Dagua, A. (1998). Guambianos. Hijos del aroiris y del agua. Bogotá, Colombia: CEREC, Editorial Los Cuatro Elementos, Fundación Alejandro Ángel Escobar, Fondo Promoción de la Cultura - Banco Popular.

Velandia Díaz, D. (2016). Biografía de las artes gráficas en Bogotá: antropología de las transformaciones del trabajo en la era neoliberal. Bogotá, Colombia: Tesis Doctoral en Antropología. Universidad Nacional de Colombia.

Velandia Díaz, D. (2007). Etnografías como libros: revisando tres productos (exitosos). Bogotá, Colombia: Tesis de Maestría en Sociología. Universidad Nacional de Colombia.

Wacquant, L., (2007). Parias urbanos: marginalidad en la ciudad a comienzos del milenio. Buenos Aires, Argentina: Manantial.

Woolgar, S. (1996). La reflexividad es el etnógrafo del texto. Cuadernos del seminario. 2 (1-2). pp. 17-35. 